\title{
Video Article \\ Event Related Potentials (ERPs) and other EEG Based Methods for Extracting Biomarkers of Brain Dysfunction: Examples from Pediatric Attention Deficit/ Hyperactivity Disorder (ADHD)
}

\author{
Geir Ogrim $^{1,2,3}$, Juri D. Kropotov ${ }^{4,5}$ \\ ${ }^{1}$ Neuropsychiatric Team, Åsebråten Outpatient Clinic, Østfold Hospital Trust \\ ${ }^{2}$ Institute of Psychology, Norwegian University of Science and Technology \\ ${ }^{3}$ Gillberg Neuropsychiatry Centre, University of Gothenburg \\ ${ }^{4} \mathrm{P}$. Bechtereva Institute of the Human Brain, Russian Academy of Sciences \\ ${ }^{5}$ Department of Neuropsychology, Andrzej Frycz-Modrzewski Krakow University \\ Correspondence to: Geir Ogrim at geir.ogrim@so-hf.no
}

URL: https://www.jove.com/video/60710

DOI: doi:10.3791/60710

Keywords: Neuroscience, Issue 157, biomarker, predictions, event-related potentials, latent components, cued GO/NOGO task, Attention Deficit/ Hyperactivity Disorder (ADHD)

Date Published: 3/12/2020

Citation: Ogrim, G., Kropotov, J.D. Event Related Potentials (ERPs) and other EEG Based Methods for Extracting Biomarkers of Brain Dysfunction: Examples from Pediatric Attention Deficit/Hyperactivity Disorder (ADHD). J. Vis. Exp. (157), e60710, doi:10.3791/60710 (2020).

\section{Abstract}

Neuropsychiatric diagnoses like ADHD are based on subjective methods like interviews, rating scales and observations. There is a need for more brain-based supplements. Stimulant medication is the most common treatment for ADHD. Clinically useful predictors of response have so far not been reported. The aim of this paper is to describe the EEG based methods we apply to extract potential biomarkers for brain dysfunction. Examples relate to biomarkers for pediatric ADHD, and prediction of medication response. The main emphasis is on Event Related Potentials (ERPs).

A nineteen channel EEG is recorded during a 3 min eyes-opened task, a 3 min eyes-closed task, and a 20 min cued visual GO/NOGO task (VCPT). ERPs are recorded during this task. The goal of the ERP protocol is to extract biomarkers of assumed brain dysfunctions that significantly differentiate between a patient group and healthy controls. The protocol includes recording during standard conditions and artifact correction. ERP waves can be used or transformed into latent components. The components of the patient group are compared with controls, empathizing components that, when compared, show relatively high effect sizes. Sub-groups of the patients are selected on the basis of the cluster analysis in the space of the components. Treatment procedure (such as medication, tDCS or neurofeedback protocol) can be applied and the changes in components related to treatment in the subgroups are observed, forming the basis for clinical recommendations.

The methods described were applied in a study of 87 pediatric ADHD patients. The index of medication response discriminated significantly between responders and non-responders with a large, and clinically meaningful effect size $(d=1.84)$. In an ongoing study comparing ADHD children with matched controls, several variables discriminate significantly between patients and controls. The global index will exceed $d=.8$. The EEG based methods described here could be clinically meaningful.

\section{Video Link}

The video component of this article can be found at https://www.jove.com/video/60710/

\section{Introduction}

In 2008, initiated by NIMH, the Research Domain Criteria (RDoC) project ${ }^{1}$ was published, aiming to find a biologically valid framework for the understanding of mental disorders. In 2013, the U.S. Food and Drug Administration (FDA) approved of the first EEG-based biomarker of ADHD to help assess ADHD in patients from 6 to 17 years of age. The Neuropsychiatric EEG-Based Assessment Aid (NEBA) System records EEG for $15-20 \mathrm{~min}$. It is based on the computation of the theta/beta ratio found to be higher in children and adolescents with ADHD than in typically developing children ${ }^{2}$. Recent publications find that this ratio does not captures all ADHD $^{3}$.

A large number of publications in clinical neuroscience demonstrate that impaired cognitive control represents a common feature of many psychiatric disorders including ADHD, schizophrenia, depression, and $O C D^{4,5}$. Theoretically, cognitive control consists of hypothetical operations allowing people to adjust flexibly to goals and contexts. Two different categories of cognitive control, proactive and reactive control, have been described $^{6}$. Our primary focus is on the reactive mode of cognitive control. Proactive cognitive control includes working memory (i.e., maintaining sensory and motor events for seconds). Reactive cognitive control includes monitoring, detection of conflict ${ }^{7,8}$, and action inhibition (for review see $\left.{ }^{9,10}\right)$. 
The GO/NOGO paradigm is sensitive to cognitive control ${ }^{11,12,13,14,15}$. GO stimuli elicit positive fluctuations from parietal brain areas. (P3 GO). The anteriorly distributed positive N2 and P3 NOGO waves, elicited by NOGO stimuli, are associated with detection of conflict and action inhibition $^{16,17,18,19}$. The N2 wave has been understood as an indicator of inhibition of action, but updated research shows that the N2 wave is associated with infrequent GO stimuli and detection of conflict ${ }^{20}$. Action inhibition is linked to the P3 NOGO wave at frontal-central sites.

The N2/P3 dichotomy may not be correct. It has been questioned by a view that ERP waves, in particular those representing cognitive control, are sums of several sources that may overlap in locations and time ${ }^{14,21}$

To disentangle the sources of ERP waves, several methods of blind source separation have been used ${ }^{15,22,23,24}$. In studies at the Institute of the Human Brain, St. Petersburg, N2d NOGO wave has been decomposed. Hidden components were detected. These components had distinct topographies and functional meanings. Only one of them was associated with detection of conflict ${ }^{14,15,25,26}$. In most adult studies of ADHD, P3 NOGO is smaller in comparison to matched healthy controls $27,28,29,30,31,32$.

The brain operations taking place during tasks of cognitive control do not seem to be correctly explained by the N2/P3 dichotomy when ERPs in GO/NOGO paradigms are analyzed ${ }^{14,15}$. Several approaches aiming to disentangle hidden components from ERP waves have been used (for review see ${ }^{21}$ ). Some studies have used independent component analysis (ICA) for ERPs in patient groups such as patients with schizophrenia ${ }^{29}$, and adults with $\mathrm{ADHD}^{33,34}$, attempting to discriminate patients from controls without diagnoses.

In (Yeredor, $2010,{ }^{25}$ p.75), a new method is suggested and adapted for ERPs. It is a method of blind source separation, based on a procedure of joint diagonalization of cross-variance matrixes. To study the functional meanings of such latent components applying this method in the cued GO/NOGO paradigm, a study from the Institute of the Human Brain was recently implemented ${ }^{26}$. In this study the action inhibition operations and the conflict detection operations were independently manipulated by modifications of the cued GO/NOGO task. A hidden component, thought to reflect detection of conflict, was found. A N2-like response and frontal topography characterized this component ${ }^{35}$. In trials requiring inhibition of prepared actions a central topography and P3-like response was seen.

In this publication the studies reported have used the traditional ERP method. Application of ICA, or the procedure of joint diagonalization of cross-variance matrixes ${ }^{25}$ (page 75) has so far not been done. In general, the results based on the different methods agree with each other, but the methods for discovering latent components seem to be more purely associated with distinct neuropsychological functions. The aim of this paper is to offer a detailed description of the WinEEG method. The focus is on ERPs, but EEG spectra and behavioral data from the GO/NOGO task are also included in the studies described to illustrate the WinEEG method.

\section{Protocol}

The equipment described in the protocol is ethically approved by the hospital authorities and is used for clinical purposes. The Regional Committee for Medical Research Ethics approved the projects described.

\section{Hardware and software for ERPs recording}

1. Use amplifiers (e.g., Mitsar 201) for EEG amplification according to manufacturer's protocol. WinEEG software and two interconnected computers are needed: the main computer for EEG and button pressing recording, and the slave computer, controlled by the main computer, for stimulus presentation.

2. Use caps with nineteen electrodes placed on the head in accordance with the international 10-20 system. NOTE: The equipment needed, caps, electrodes, gel etc. can be obtained from several manufacturers.

3. Place patients/subjects in a comfortable chair in a sound-proof room with no backlight.

4. Use referential montage (Ref) which is default. For later comparisons with the HBi database; change montage to common average (Av).

\section{Competence and education}

1. Ensure that everyone involved in registration of EEG, interpretation of data, clinical use and research must have the necessary professional background and specific training in the use of WinEEG.

\section{Informing patients/participants}

1. Inform the patients/subjects that the procedure is not dangerous, and it does not hurt. NOTE: The syringe needed to fill the 19 holes with gel is not sharp.

2. Inform the children that the researchers cannot read their minds. Tell them that in every brain some electrical activity takes place. To read it, the researchers need this equipment. There is nothing with the brain, but the researchers want to see how it works when it relaxes, and when it must attend to a task.

3. Leave the equipment in place for $10 \mathrm{~min}$. NOTE: During this time period, the youngest children can watch a cartoon if they want. Sometimes a parent is silently present in the back of the room until the child feels safe.

4. Inform the subject that the test takes about one hour. When the equipment is properly placed, tell the subject to relax with closed eyes for 3 $\min$, followed by 3 min relaxed with open eyes. 


\section{Creating the EEG data files}

NOTE: WinEEG has its own build-in databases separately for storing raw EEG files (extension - .eeg), EEG spectra (extension - .spec), and ERP files (extension - .erp). The databases are created automatically and initially stored in WinEEG/data, WinEEG/spec and WinEEG/erp folders.

1. To start a session with a patient, create a corresponding EEG data file by clicking on menu File | New. The patient's card appears on the screen.

NOTE: The following format is used: Put in the name or the code of the person to be tested. Put in the date of birth (DD.MM.YYYY), and M or $F$ for sex. Use the other open fields on the patient card as needed.

\section{Preparation of the equipment}

1. Use the small color-coded band to measure the circumference of the head to find the right size of the cap. Use a small patch to wipe off oil from earlobes and forehead. Measure the nasion-inion distance $(e x .35 \mathrm{~cm})$. The center of the frontal pole electrodes should fall on a horizontal line $10 \%$ of this distance above the nasion $(\mathrm{ex} .3 .5 \mathrm{~cm})$.

NOTE: The patient's hair must be clean (without hair products, etc.) and dry.

2. Put some skin prep gel (e.g., Neuprep) on the earlobes. Fill the ear electrode cups with conductive electro-gel (e.g., Ten20) and put on the ear-electrodes. Make sure that the other ends of these cables are connected to the amplifier.

3. Tell the subject that the earlobe electrodes capture the "noise-electricity" in the room, and that this is subtracted from the electricity from the head to see what comes from the head without noise.

4. Place the cap symmetrically on the head with the frontal pole electrodes placed as described above. Pull the cap down as far as possible to secure that it is close to the head.

5. Put the waistband around the chest and fasten the push buttons connected to the cap to this band. This will prevent movements of the cap and electrodes during testing.

6. Connect the cap-cable and the ear cables to the amplifier. Do not mix the left and right ear.

7. Click on the WinEEG menu control of impedance icon. A simplified head with all electrodes appears. Second, fill the syringe with gel and use it to fill all electrode holes. Impedance is OK when the black circles are yellow. NOTE: Fill all the holes before starting work with each electrode to secure proper impedance. Use a thin wooden stick to help the gel reach the skin of the head, which is a prerequisite for capturing electrical brain activity.

8. Start registration when all 20 holes have a yellow color on the screen, and the color difference between individual holes is small.

9. Start monitoring the EEG record. Click on the green arrow (EEG monitoring). NOTE: When the raw EEG signals from all 19 electrodes are seen, ask them to blink their eyes and point out the large changes at frontal sites. These artefacts can be automatically removed. The quality of the registration depends on relaxed jaws, eyes and forehead, and that all kind of head and body movements must be avoided as much as possible.

\section{Registrations of "eyes closed" and "eyes opened"}

1. Tell the subject to relax in the comfortable chair with eyes closed for $3 \mathrm{~min}$. Before starting "eyes opened" tell them to sit quiet for $3 \mathrm{~min}$ with their eyes opened resting on the screen in front of them.

2. Push the red circle (acquire EEG) on the menu line, next to the "control of impedance" button.

3. Choose "eyes closed" or "eyes opened" by pushing the next icon drop-down menu. This will start the registration. The elapsed time in seconds can be seen above the EEG curves.

4. Push the pause-button (not "save" as this will make further registrations in the same file impossible) after $180 \mathrm{~s}$. Ask them to open their eyes (or close them if eyes opened was the first part). Continue the registration. After 3 min, stop and save. NOTE: If not saved, data will be lost when creating a new file.

\section{Preparations for EEG recordings in the cued GO/NOGO task}

1. Insert a special USB dongle in the proper port before starting an EEG recording. A small light on the dongle indicates that the system is working.

2. Give the subject a special button-switch in his/her right hand. It is needed to complete a test that takes about 20 min.

3. Open the task menu and choose VCPT on the slave computer in front of the patient/participant. NOTE: An instruction window showing all four combinations of pictures appears on the slave computer screen: (animal-animal (a-a), animalplant (a-p), plant-plant ( $p-p)$ and plant-human ( $p-h)$. (Figure 1). The stimuli are presented on a 17-inch computer screen, 1.5 meter in front of the subjects. The sound measured at the subject's head must be $70 \mathrm{~dB}$.

4. Instruct the subject that the pictures come in pairs, the first picture is followed by the second one in $1 \mathrm{~s}$. After $3 \mathrm{~s}$ a new pair will start.

5. Tell the subject that the left mouse button must be pressed every time the a-a combination appears. In a-a combinations, the two animals are always identical.

6. Inform the subject to be as precise as possible, but also fast. The subject should not push the mouse button on a-p, p-p or a-h combinations.

7. Tell the subject to ignore the random sounds in $\mathrm{p}-\mathrm{h}$ combinations.

8. Press enter on the slave computer and run the task for about 2-5 min to train the subject until he/she understands properly.

9. On the slave computer choose the switch to slave mode option. NOTE: The task presentation is now managed by the WinEEG program on the main computer. 


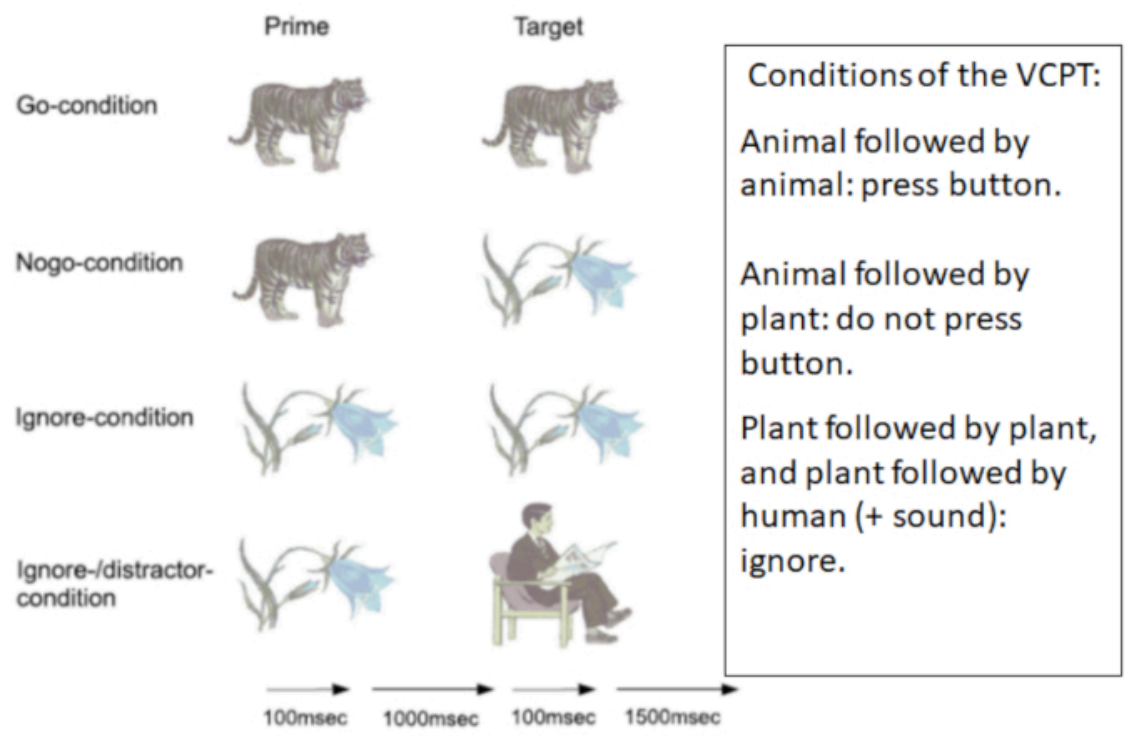

Figure 1: VCPT: Visual Continuous Performance Test. Figure 1 shows the four conditions of the VCPT. One hundred trials of each condition are presented randomly. The total test time is $20 \mathrm{~min}$. Please click here to view a larger version of this figure.

\section{EEG and button press recordings in task condition}

1. Start the EEG Monitoring option on the main computer from recording menu to check that the system works properly.

2. Start EEG acquisition option from recording menu on the main computer menu box. Stimuli presentation program option is highlighted. Choose VCPT and start the stimuli presentations on the slave computer.

NOTE: Every time the left mouse button is pressed, a red mark is seen beneath the EEG curves. There are two lines, one for the left and one for the right mouse button.

3. Check that the red marks do not appear on the lowest line, as this indicates they used the right button. Use the pause button and tell the subject to use the left mouse button.

4. Monitor the EEG registration and note incidents of potential interest. If too many artifacts, related to muscles or movements, are observed; pause the registration and remind the subject to relax and sit still. Continue registration.

NOTE: The EEG registration during the task takes 20 min and consists of 400 pairs of pictures; 100 of each pair; a-a, a-p, p-p and p-h. The pairs are presented in random order. (See Figure 1).

5. Take a short break after every 100 pairs. Push the pause button. When sitting in the chair encourage them to stretch their arms and legs and loosen their face muscles. Offer something to drink. Continue the registration after a minute or two.

\section{Ending the registration}

1. Stop the registration when all 400 trials are completed by pressing Recording | Stop. NOTE: By pressing the File Save option, the file with the recorded raw EEG and button pressing channel will be stored in the built-in database.

2. Tell the subject to remain seated until the researcher removes the cap, waist band, and ear electrodes. NOTE: Usually there is no gel in the hair when this is completed. Remove gel with a paper cloth. Most patient/participant prefer to wash their hair when they are back home, but there should be an option to wash their hair right after the registration.

3. Thank the subject for completing the task. Ask them to rate the VCPT (1-10 scale), How boring? How exhausting? Depending on the agreement, tell them what happens next regarding the test results.

\section{Cleaning}

1. Clean the ear electrodes (dip in hot water), and cap as soon as possible. Water, soap and a soft brush are needed. Check all holes to see that gel leftovers are removed before the caps are hung somewhere to dry.

\section{Preprocessing the EEG record}

NOTE: Three different electrode montages are provided in the HBIdb software. They are: linked ears reference (labeled as Ref), common average reference (labeled as Av), and local average reference (labeled as Aw). Select montage from Montage list in SETUP menu. EEG is recorded in Ref. Change to Av before starting artifact correction.

1. Remove eye movement artifacts by applying a procedure of spatial filtration. NOTE: The spatial filters are obtained by application of Independent Component Analysis (ICA) to a selected EEG fragment. The fragment must contain at least $90 \mathrm{~s}$ of raw data. 
2. Select the EEG fragment by clicking the left button of the mouse on the time bar at the beginning of the fragment and by clicking the right button on the time bar at the end of the fragment. The whole fragment after the selection is highlighted by yellow color.

NOTE: The artifact correction procedure is implemented by choosing the Artifact correction option from Analysis menu. The window named Spatial filter parameters estimation appears on the screen.

3. Select the ICA method to be able to compare the individual spectra and ERPs with the HBI reference database.

NOTE: The Spatial filters parameters estimation window appears. From the left to the right: 1) a fragment of raw (uncorrected) multi-channel EEG, 2) a fragment of corrected EEG, 3) waveform of corrected artifacts, 4) time courses of extracted ICA components (from the top to the bottom) and 5) topographies of the independent components.

4. Select the topographies associated with eye blinks and horizontal eye movements. Accept by clicking OK.

5. Implement the procedure named Mark artifacts by clicking the Mark artifacts option from the Analysis menu.

NOTE: This will reject slow and high frequency fragments-outliers: EEG fragments that are contaminated by non-eye movement artifacts (episodes of muscle tension, head movements etc.) are excluded from further analysis. In some complex cases manual artifact rejection can supplement the procedure described.

\section{Computing EEG spectra}

1. Choose EEG spectra and fragment- eyes closed or eyes opened from the Analysis menu. If default settings are as shown in Figure 2 press OK.

NOTE: Spectra are shown in Figure 3 for all 19 sites. The $x$-axis is frequency in Hertz $(\mathrm{Hz})$ and the $y$-axis is power in microvolt $(\mu \mathrm{V})$.

2. Choose Analysis | Comparison of results to compare the individual spectra with the database. In the left window choose the patient/subject. In the right window choose the database file (or another opened file) and press OK for comparison. An image showing the difference between the subject and the database appears.

NOTE: Spectra show the squared mean power of the frequencies from $0 \mathrm{~Hz}$ to $30 \mathrm{~Hz}$. (Frequencies below $1.5-2 \mathrm{~Hz}$ should be interpreted with caution as they are often influenced by artifacts). To interpret spectra is beyond the scope of this protocol. Paroxysmals of potential clinical interest may not appear in spectra but can be seen in raw EEG.

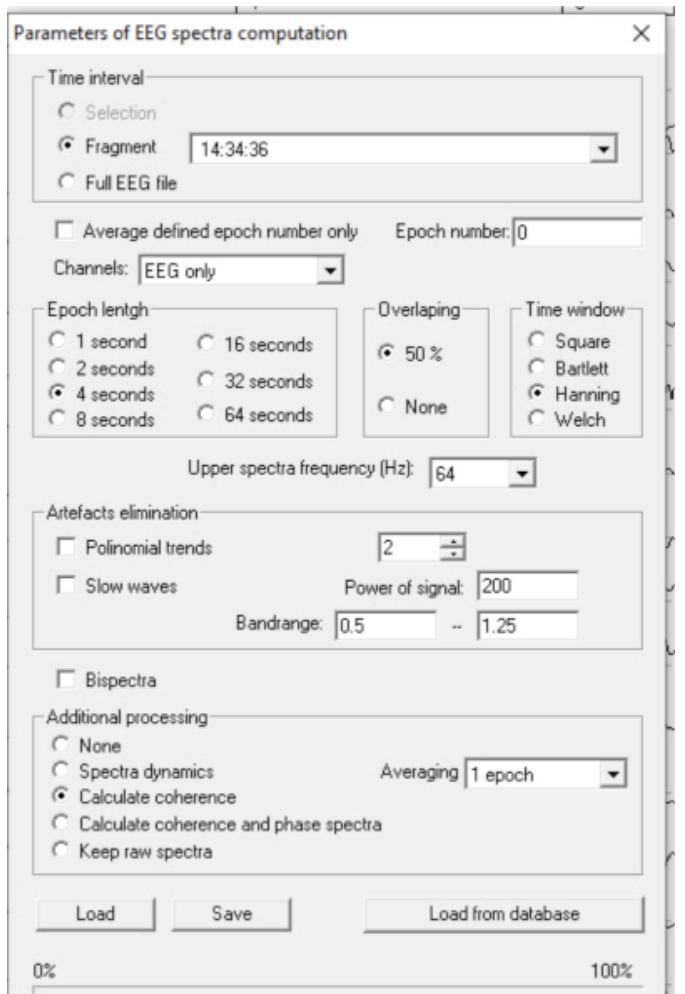

Figure 2: Computing EEG spectra. To compute spectra: Click Analysis | Spectra. If the settings are correct, the picture shown in Figure 2 appears. Please click here to view a larger version of this figure. 


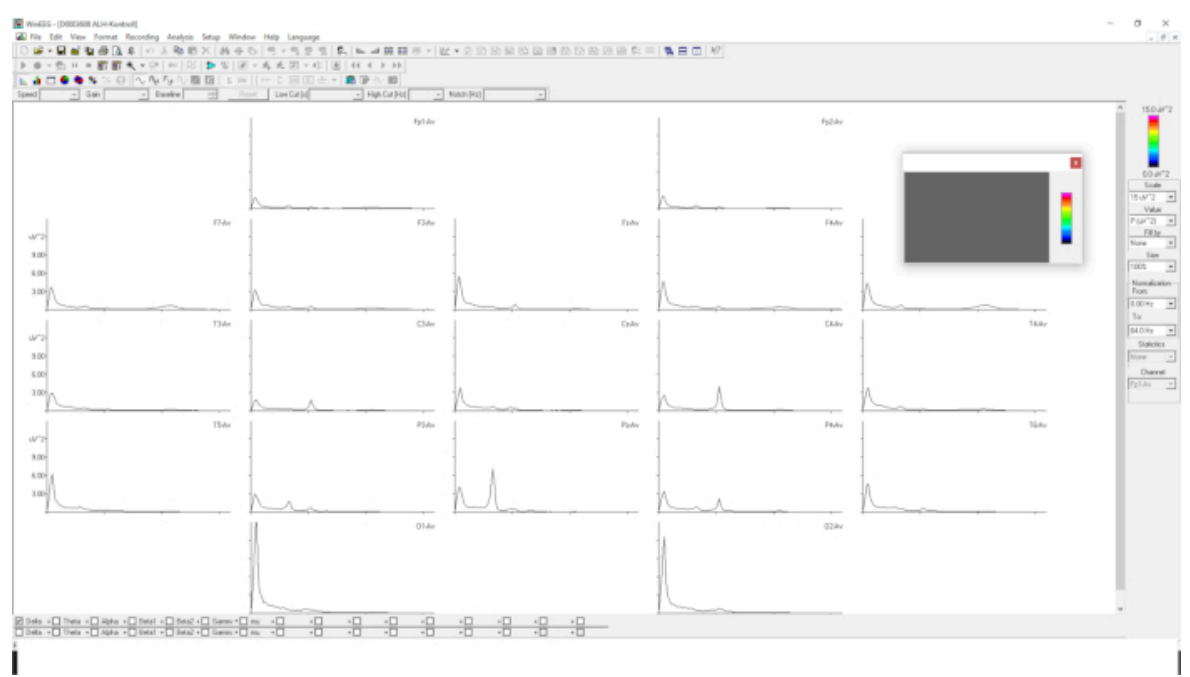

Figure 3: EEG spectra with 19 electrodes. Figure 3 shows EEG spectra in 19 sites. The $\mathrm{x}$-axis is frequency from $0-30 \mathrm{~Hz}$. The $\mathrm{y}$-axis is power in $\mu \mathrm{V}^{2}$. Please click here to view a larger version of this figure.

\section{Computing Event Related Potentials (ERPs)}

NOTE: Event Related Potentials (ERPs) are computed by an averaging procedure. Only correct trials are included. ERPs are computed after completion of the preprocessing described above. The gold standard for computing ERPs is to keep the number of the averaged trials above 50 .

1. Select an EEG VCPT file in common average montage (Av). Click Compute ERP from the Analysis menu.

NOTE: If the HBdb dongle is not connected to the computer or is not activated for some reason, the HBdb allows the user to read the EEG files and to preprocess them but does NOT allow to compute spectra and ERPs with the database.

2. Click OK. A window depicting parameters of ERP computations occurs (Figure 4).

NOTE: As shown in Figure 4, the parameters are as follow: Trial groups: 1: a-a GO; 2: a-p NOGO; 3: p-p (ignore trials); 4: p-h (novelty trials); as well as 5 : + meaning all trials started with presentation of a; and $6:-$ meaning all trials started with presentation of $p$. In addition, the ERP difference waves are shown.

3. Click OK. The ERPs for the analyzed file are computed and presented in graphical form in the ERP window (Figure 4).

NOTE: The ERP window consists of 19 graphs of ERPs for each task condition: a-a GO, a-p NOGO, p-p, p-h. Only a-a GO and a-p NOGO are shown in Figure 4. In each graph: X-axis is time in ms., Y-axis is voltage in $\mu \mathrm{V}$. Figure 4 presents the results of adjustment of the presentation form. Only categories of trials a-a GO, a-p NOGO are selected (GO is depicted in green color, NOGO is depicted in red color). The scale is selected at $12 \mu \mathrm{V}$. The time interval is selected from $1400 \mathrm{~ms}$; the time of presentation of Stimulus 2 is selected with a duration of $700 \mathrm{~ms}$.

4. To map the wave for Event Related Potentials (ERPs) click the right button of the mouse at the selected time (along $X$-axis) on any graph, then release it and select Add map option for the opened menu. The corresponding maps appear at the bottom of the page.

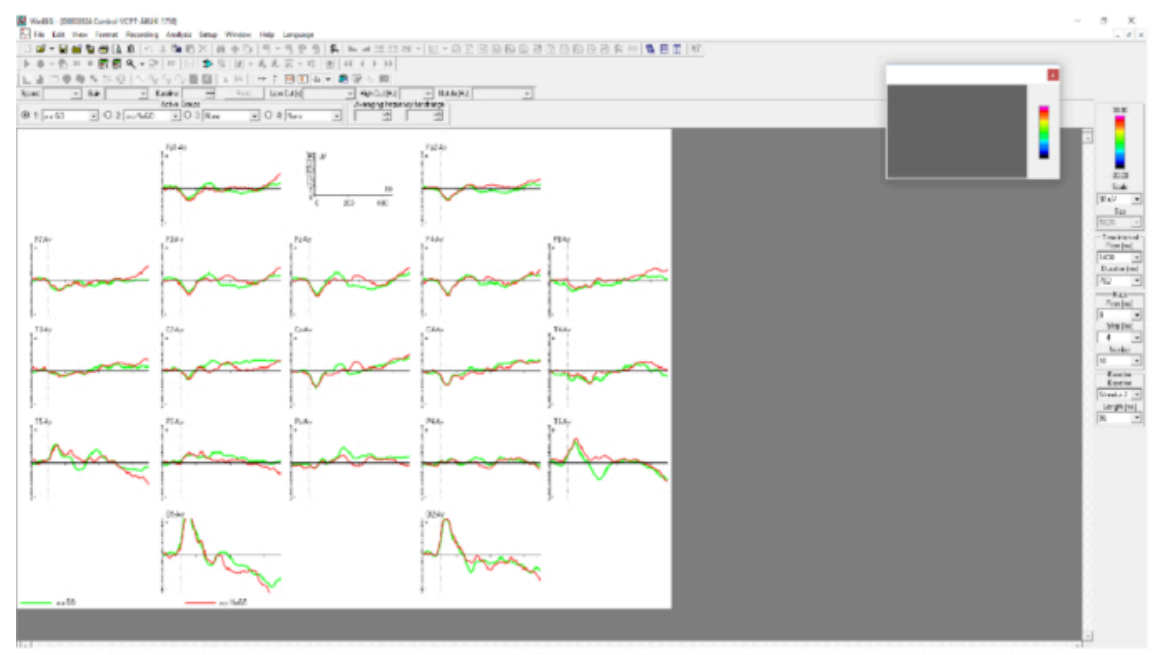

Figure 4: Parameters of ERP computations. Figure 4 shows ERP components a-a GO (green) and a-p NOGO (red) in 19 sites. The time interval is $1400 \mathrm{~ms}$ to $2100 \mathrm{~ms}$. A-a GO is most clearly seen at site Pz and a-p NOGO at Cz. Please click here to view a larger version of this figure. 


\section{Registration and comparison of behavioral data in VCPT}

1. Click on the right mouse button in the window showing ERPs and choose groups info to see number of omissions (missed button clicks), commissions (impulsive extra clicks, mean reaction time (RT) for a-a-GO, and RT variability.

2. Choose Analyses | Comparison of results. Mark the subject's file in the left window and the comparison file in the right window. Click OK NOTE: We use age-based paper-tables showing the percentiles for these variables in the norm group.

\section{Comparing Event Related Potentials (ERPs) with the reference data base}

NOTE: The time interval of interest for comparison is defined by typing the corresponding numbers in the menu: Time interval from (ms), Duration (ms). ERPs can be selectively presented for certain categories of trials (such a-a GO, a-p NOGO, p-p, p-h) by selecting the corresponding graph from the menu Active groups on the top of the ERP window.

1. Choose Comparison of results from the Analysis menu. Click on the patient/subject in the left window and click on the HBIdb in the right window.

NOTE: To compare the subject with for example a previous registration, open the old file and choose this instead of HBIdb). The ERP comparison window appears. This window depicts the difference ERP wave in $\mu \mathrm{V}$. Subject-Mean Reference is computed for each channel.

2. Press the right button of the mouse at a time point of interest and at a channel of interest in order to obtain the significance level of deviation from the reference. The corresponding values appear at the bottom of the window showing the difference in $\mu \mathrm{V}$, the time point selected and the p-value.

NOTE: Presenting difference waves with confidence levels is an available option. The blue line under the graphs depicts confidence levels of deviation from the reference computed for each channel and each time point for the Group 3.

3. Select groups for ERP difference by clicking the arrow at 1 (the individual's ERPs). Select a-a GO [1]. For referential ERPs click 2. Select a-a $G O$ [2]. Click 3 and a-a GO [D] to see the difference (1-2). Active group 4: Select None from this menu.

4. Define the time interval of interest by typing (or selecting) the corresponding numbers in the menu: time interval from (ms), duration (ms) (e.g., 1400, 700 to analyze ERPs in response to the second stimulus).

5. Put the cursor at a time point and channel of interest and click the right button of the mouse and select Add map from the pop out menu. A map showing the deviance from the reference is shown.

NOTE: Comparing independent components of Event Related Potentials (ERPs) with the reference data base, the software provides an option of decomposing individual ERPs into independent components. The components are associated with distinctive psychological operations.

\section{Representative Results}

\section{Prediction of medication response in pediatric ADHD}

ADHD is a common neuropsychiatric childhood disorder ${ }^{36}$. It is characterized by symptoms of inattention accompanied by symptoms of hyperactivity and impulsivity. Impairment in school, home, and leisure settings are common. In school aged children, the estimated prevalence is $5 \%$ to $7 \%$. Comorbidities are common. Medical treatment, using stimulants based on methylphenidate (MPH) or dextroamphetamine (DEX), are widely used. Positive effects of stimulant medication (reductions in restlessness, hyperactivity and impulsivity and improved attention) are reported in $70 \%$ of the patients. Shifting from medication based on MPH to DEX can increase positive effects to $80 \%{ }^{37,38}$. Frontal-striatal circuits seem to be activated by stimulants ${ }^{39}$.

There is no generally accepted definition of a medication response that is clinically meaningful. Applying rating scales, comparing baseline scores with scores on medication, is the most commonly used method. In some studies, a $25 \%$ or $50 \%$ reduction of scores is used as definition of response. In other studies, scores not exceeding 1 SD above population mean are used ${ }^{40,41}$. Clinically, an overall decision based on all relevant available data is used. To evaluate side effects, such as loss of appetite, insomnia, increased irritability, or anxiety, is important ${ }^{37,42}$

The use of rating scales can be criticized for several reasons. Small correlations $(0.30-0.50)$ between teacher and parent scores are reported in several studies ${ }^{4}$. The search for clinically useful predictors of response is motivated by a large number of non-responders, informants that do not agree, and the fact that everyone can have some modest effects of improved attention when small doses of stimulants are used. Published research on predictors of response include ADHD subtype, demographics, comorbid disorders, gene variables, scores on rating

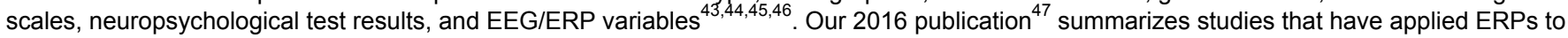
predict medication response.

In previous studies, we analyze d data from the cued visual GO/NOGO task (i.e., attention test data, EEG spectra, and ERPs). In one study, we found 3 variables contributing significantly to the prediction of side-effects. These variables were combined to an index that was considered clinically meaningful ${ }^{42}$. In a study on clinical effects, applying the same methods, the prediction index was also considered clinically useful ${ }^{48}$. The effects of a single dose of stimulant medication on medication responders (REs) and non-responders (non-REs) was investigated in a third study ${ }^{47}$. The test procedure was completed twice, the first test with no medication, and the second test an hour after having received a trial dose. Based on rating scales and interviews after a 4-week medication trial, the patients were classified as REs or non-REs. Our focus was on changes in cognitive ERPs and attention test scores. We found that the effects on the P3 NOGO component was significantly different in the two groups, with a large effect size $(d=1.76)$. A significant increase of the component amplitude was seen in REs but not in non-REs. Predictions of response based on two tests was improved compared with predictions based only on test 1. 
In our latest study, we developed two global indexes, one for prediction of clinical gains and one for prediction of side-effects. As described above we combined variables that discriminated significantly between compared groups with a modest or large effect size. Each variable was weighted in accordance with the effect size. We examined variables from all three WinEEG domains: EEG spectra, ERPs and behavior. The following variables were combined: Test 1: P3NOGO amplitude and theta/alpha ratio; differences between Test 2 and Test 1: Omission errors, reaction time variability, contingent negative variation (CNV) and P3NOGO amplitude. The effect size of the global scale was $d=1.86$. Accuracy was 0.92 . Prediction of side-effects was based on 4 variables: Test 1: RT, Test 2: novelty component, alpha peak frequency, and reaction time changes (Test 2 - Test 1). The global scale $d$ was 1.08 and accuracy was $0.78^{49}$.

\section{Some preliminary results}

In an ongoing study, we compare a group of 61 ADHD patients age 9-12 years and a group of 67 age-matched healthy controls (HC). The final statistical analyses have so far not been completed. Below we are presenting the preliminary results obtained from WinEEG assessment.

Behaviorally, the ADHD group showed an inattention pattern with statistically (at $\mathrm{p}<0.001$ ) more omission errors in comparison to the healthy controls (HC) group ( $13.7 \%$ vs. $4.8 \%)$ accompanied by an attention lapses pattern expressed in statistically higher $(p<0.001)$ variability of reaction time (151 ms vs. $125 \mathrm{~ms})$.

The main results of comparing ERP waveforms between the two groups are shown in Figure $\mathbf{5}$ and Figure $\mathbf{6}$. Figure $\mathbf{5}$ demonstrates the ERP correlates of dysfunction of proactive cognitive control in ADHD group. Two indexes of proactive cognitive control (P3 cue wave and CNV wave) are reduced in the ADHD group in comparison to the HC group. Figure 6 demonstrates the ERP correlates of dysfunction of reactive cognitive control in the ADHD group. Two indexes of reactive cognitive control (N2 NOGO and P3 NOGO) are reduced in the ADHD group in comparison to the $\mathrm{HC}$ group.

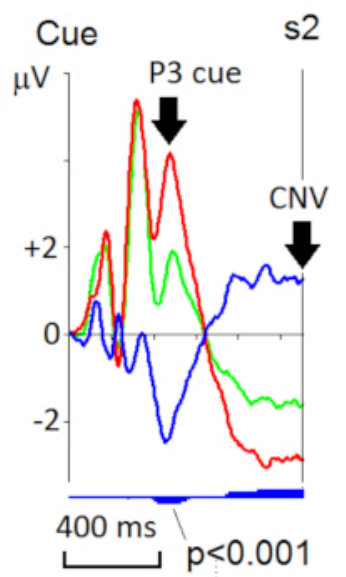

(a)
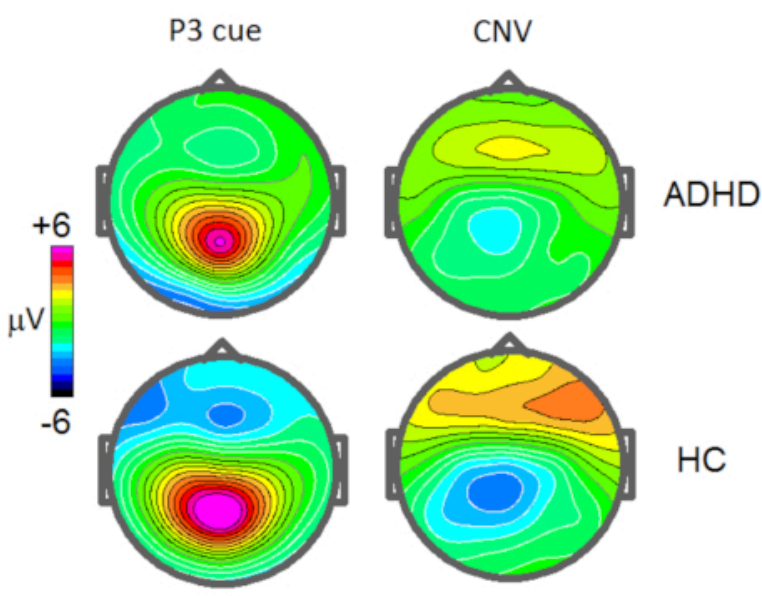

(b)

Figure 5: Grand average ERP wave patterns (a) and the corresponding maps (b) in proactive cognitive control in ADHD and healthy control (HC) groups. (a) ERPs measured at P3 in ADHD group (green line) and HC group (red line) and their difference (ADHD-HC) wave (blue line). Blue vertical bars below the curves indicate level of statistical significance of the difference (small bars - $p<0.05$, middle bars - $p<0.01$, large bars - $p<0.001$ ). Arrows indicate the classical waves - P3 cue and CNV (contingent negative variation). (b) Maps at the maximums of amplitudes of $\mathrm{P} 3$ and $\mathrm{CNV}$ waves for the two groups. Please click here to view a larger version of this figure. 


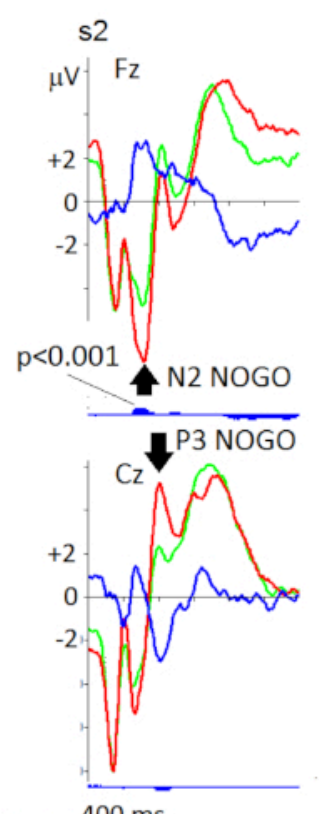

(a)

$400 \mathrm{~ms}$

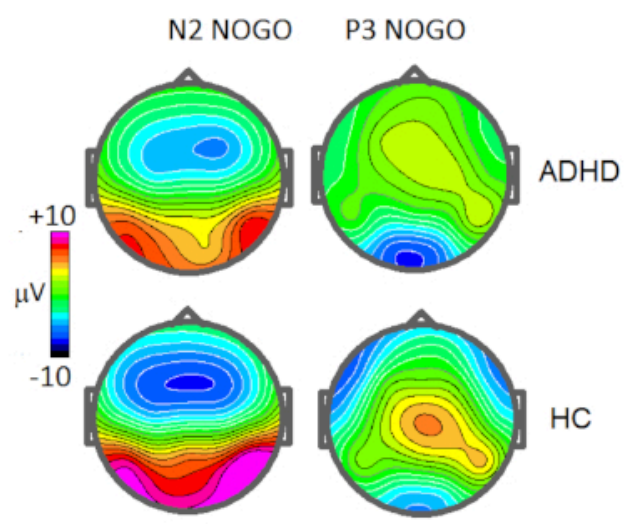

(b)

Figure 6: Grand average ERP wave patterns (a) and the corresponding maps (b) in reactive cognitive control in ADHD and healthy control (HC) groups. (a) ERPs measured at Fz and Cz ADHD group (green line) and HC group (red line) and their difference (ADHD-HC) wave (blue line). Blue vertical bars below the curves indicate the level of statistical significance of the difference (small bars - $p<0.05$, middle bars $p<0.01$, large bars $-p<0.001$ ). Arrows indicate the classical waves - N2 NOGO and P3 NOGO. (b) Maps at the maximums of amplitudes of N2 NOGO and P3 NOGO waves for the two groups. Please click here to view a larger version of this figure.

As one can see the ADHD group shows hypo-functioning of multiple operations of cognitive control. These operations occur in different time windows and in different spatial locations. A particular patient might have only one hypo-functioning indicating the source of the individual disorder and the ways of its correction.

\section{Clinical significance}

To compute a clinically useful biomarker for a heterogenous diagnosis such as ADHD, several variables that differ significantly between ADHD and controls need to be combined. The effect size $(d)$ of an index should be above $d=.8$. An important next step will be applying this index when ADHD is compared with clinical controls.

\section{Discussion}

Diagnoses in psychiatry are based on observed behavior. In most cases, a specified number of symptoms must be observed in different settings for 6 months or more. An important part of the diagnostic process is to exclude somatic etiology. In addition, other psychiatric diagnoses must be considered. Quite often the symptoms of interest can be part of another diagnostic category. If several symptoms overlap with other disorders, the clinician must decide if this second disorder is a comorbid or differential diagnosis.

The clinical tools available are diagnostic interviews, rating scales, medical and developmental history, psychological tests and direct observations. Most of these methods are quite subjective; heavily influenced by the informant as well as the professional. Rating scales from parents and teachers usually show quite modest correlations $(r=0.3-0.5)$.

In the representative results, we argue that the underlying mechanisms in ADHD probably differ from patient to patient. Lack of (language) understanding, problems with self-motivation, sensitivity to external distractors, etc. can all lead to symptoms of inattention. The EEG based methods described in this paper can help solve some of these challenges. The problem of subjective interpretations is absent. The ERP methods described seem to reveal underlying psychological operations like working memory, action inhibition, monitoring, response preparation, etc. involving specific brain structures. Deficits in these mechanisms are not limited to specific diagnostic categories. We believe that in the future, treatment (medication, neurofeedback, cognitive training, tDCS, ...) will focus on such cognitive and/or emotional operations and their underlying brain mechanisms and not on the current diagnostic categories.

A purpose of a diagnosis is to determine the best treatments. To evaluate the effects of treatment, self-reported and observed improvements are of course decisive. Such reports can to some degree represent placebo effects, however, and should be supported by (partial) normalization of underlying brain dysfunctions reflected in for example changes in ERP components. This combination of subjective and objective measures of treatment effects are important in both clinic and research. 
For reasons as those mentioned above, it is no surprise that people with the same diagnoses often do not respond to the same medical treatments. In personalized medicine diagnoses are supplemented with empirically based measures of response prediction to pinpoint the best treatment for the individual patient. In this paper we have described our research on prediction of stimulant medication response in pediatric ADHD. To find reliable predictors of positive response to antidepressant medication is perhaps even more important as the time needed to evaluate responses is long, as is the titration period. The procedures described in this paper could contribute to the ongoing EEG and ERP based research on prediction of medication effects in depression ${ }^{53}$.

The EEG based methods described are non-invasive and affordable, and well suited for research as well as for clinical work.

\section{Disclosures}

Ogrim G. has nothing to disclose. Kropotov J.D. was the head of the laboratory at the Institute of the Human Brain, St. Petersburg when and where the WinEEG program was developed. He has no economic interests in the system.

\section{Acknowledgments}

None.

\section{References}

1. Insel, T. et al. Research domain criteria (RDoC), toward a new classification framework for research on mental disorders. American Journal of Psychiatry. 167 (7), 748-751 (2010).

2. Snyder, S.M., Rugino, T.A., Hornig, M., Stein, M.A. Integration of an EEG biomarker with a clinician's ADHD evaluation. Brain and Behavior. 5 (4), e00330 (2015)

3. Arns, M., Conners C.K., Kraemer, H.C. A decade of EEG Theta/Beta Ratio Research in ADHD: a meta-analysis. Journal of Attention Disorders. 17 (5), 374-383 (2013).

4. Egeland, J. Differentiating attention deficit in adult ADHD and schizophrenia. Archives of Clinical Neuropsychology. 22 (6), 763-771 (2007)

5. Egeland, J. et al. Attention profile in schizophrenia compared with depression: differential effects of processing speed, selective attention and vigilance. Acta Psychiatrica Scandinavia. 108 (4), 276-284 (2003).

6. Braver, T.S. The variable nature of cognitive control: a dual mechanisms framework. Trends in Cognitive Science. 16 (2), 106-113 (2012).

7. Botvinick, M.M. Conflict monitoring and decision making: reconciling two perspectives on anterior cingulate function. Cognitive and Affective Behavior Neuroscience. 7 (4), 356-366 (2007).

8. Ullsperger, M., Fischer, A.G., Nigbur, R., Endrass, T. Neural mechanisms and temporal dynamics of performance monitoring. Trends in Cognitive Science. 18 (5), 259-267 (2014).

9. Bari, A., Robbins, T.W. Inhibition and impulsivity: behavioral and neural basis of response control. Progress in Neurobiology. 108 (Sep), $44-79$ (2013).

10. Huster, R.J., Enriquez-Geppert, S., Lavallee, C.F., Falkenstein, M., Herrmann, C.S. Electroencephalography of response inhibition tasks: functional networks and cognitive contributions. International Journal of Psychophysiology. 87 (3), 217-233 (2013).

11. Simson, R., Vaughan, H.G., Jr, Ritter, W. The scalp topography of potentials in auditory and visual Go/NoGo tasks. Electroencephalography and Clinical Neurophysiology. 43 (6), 864-875 (1977).

12. Falkenstein, M., Hoormann, J., Hohnsbein, J. ERP components in Go/Nogo tasks and their relation to inhibition. Acta Psychologica (Amst). 101 (2-3), 267-291 (1999).

13. Verleger, R., Gorgen, S., Jaskowski, P. An ERP indicator of processing relevant gestalts in masked priming. Psychophysiology. 42 (6), 677-690 (2005).

14. Kropotov, J.D., Ponomarev, V.A., Hollup, S., Mueller, A. Dissociating action inhibition, conflict monitoring and sensory mismatch into independent components of event related potentials in GO/NOGO task. Neuroimage. 57 (2), 565-575 (2011).

15. Kropotov, J.D., Ponomarev, V.A. Decomposing N2 NOGO wave of event-related potentials into independent components. Neuroreport. 20 (18), 1592-1596 (2009).

16. Bekker, E.M., Kenemans, J.L., Verbaten, M.N. Electrophysiological correlates of attention, inhibition, sensitivity and bias in a continuous performance task. Clinical Neurophysiology. 20115 (9), 2001-2013 (2004).

17. Fallgatter, A.J., Aranda, D.R., Bartsch, A.J., Herrmann, M.J. Long-term reliability of electrophysiologic response control parameters. Journal of Clinical Neurophysiology. 19 (1), 61-66 (2002).

18. Fallgatter, A.J., Strik, W.K. The NoGo-anteriorization as a neurophysiological standard-index for cognitive response control. International Journal of Psychophysiology. 32 (3), 233-238 (1999).

19. Bruin, K.J., Wijers, A.A., van Staveren, A.S. Response priming in a go/nogo task: do we have to explain the go/nogo N2 effect in terms of response activation instead of inhibition? Clinical Neurophysiology. 112 (9), 1660-1671 (2001)

20. Randall, W.M., Smith, J.L. Conflict and inhibition in the cued-Go/NoGo task. Clinical Neurophysiology. 122 (12), $2400-2407$ (2011).

21. Kappenman, E.S., Luck, S.J. ERP Components: The Ups and Downs of Brainwave Recordings. In: Luck, S.J., Kappenman, E.S., editors. The Oxford Handbook of Event-related Potential Components. 1st ed. Oxford: Oxford University Press; 3-29 (2012).

22. Ponomarev, V.A., Kropotov, I. Improving source localization of ERPs in the GO/NOGO task by modeling of their cross-covariance structure. Fiziol Cheloveka. 39 (1), 36-50 (2013).

23. Makeig, S., Jung, T.P. Tonic, phasic, and transient EEG correlates of auditory awareness in drowsiness. Brain Research and Cognitive Brain Research. 4 (1), 15-25 (1996).

24. Onton, J., Makeig, S. Information-based modeling of event-related brain dynamics. Progressions in Brain Research. 159, 99-120 (2006).

25. Kropotov, J.D. Functional Neuromarkers for Psychiatry: Applications for Diagnosis and Treatment. 1st ed. London: Elsevier/Academic Press (2016). 
26. Kropotov, J.D, Ponomarev, V.A., Pronina, M., Jancke, L. Functional indexes of reactive cognitive control: ERPs in cued go/no-go tasks. Psychophysiology. 54 (12), 1899-1915 (2017).

27. Bekker, E.M., Kenemans, J.L., Verbaten, M.N. Source analysis of the N2 in a cued Go/NoGo task. Brain Research and Cognitive Brain Research. 22 (2), 221-231 (2005).

28. Fisher, T., Aharon-Peretz, J., Pratt, H. Dis-regulation of response inhibition in adult Attention Deficit Hyperactivity Disorder (ADHD), an ERP study. Clinical Neurophysiology. 122 (12), 2390-2399 (2011).

29. Olbrich, H.M., Maes, H., Valerius, G., Langosch, J.M., Feige, B. Event-related potential correlates selectively reflect cognitive dysfunction in schizophrenics. Journal of Neural Transmission (Vienna). 112 (2), 283-295 (2005).

30. Grane, V.A. et al. Correction: ERP Correlates of Proactive and Reactive Cognitive Control in Treatment-Naive Adult ADHD. PLoS One. 11 (9), e0163404 (2016).

31. Woltering, S., Liu, Z., Rokeach, A., Tannock, R. Neurophysiological differences in inhibitory control between adults with ADHD and their peers. Neuropsychologia. 51 (10), 1888-1895 (2013).

32. Wiersema, R., van der Meere, J., Roeyers, H., Van Coster, R., Baeyens, D. Event rate and event-related potentials in ADHD. Journal of Child Psychology and Psychiatry. 47 (6), 560-567 (2006).

33. Mueller, A., Candrian, G., Kropotov, J.D., Ponomarev, V.A., Baschera, G.M. Classification of ADHD patients on the basis of independent ERP components using a machine learning system. Nonlinear Biomedical Physics. 4 (Suppl 1), (2010).

34. Mueller, A. et al. Discriminating between ADHD adults and controls using independent ERP components and a support vector machine: a validation study. Nonlinear Biomedical Physics. 5, 5 (2010).

35. Kropotov, J.D. et al. Latent ERP components of cognitive dysfunctions in ADHD and schizophrenia. Clinical Neurophysiology. 130 (4), 445-453 (2019).

36. Spencer, T.J., Biederman, J., Mick, E. Attention-deficit/hyperactivity disorder: diagnosis, lifespan, comorbidities, and neurobiology. Journal of Pediatric Psychology. 32 (6), 631-642 (2007).

37. Barkley, R.A. Attention - Deficit Hyperactivity Disorder. A Handbook for Diagnosis and Treatment. Third ed. New York, London: The Guilford Press (2006).

38. Spencer, T. et al. Pharmacotherapy of attention-deficit hyperactivity disorder across the life cycle. Journal of the American Academy of Child and Adolescent Psychiatry. 35 (4), 409-432 (1996).

39. Rubia, K. et al. Effects of stimulants on brain function in attention-deficit/hyperactivity disorder: a systematic review and meta-analysis. Biological Psychiatry. 76 (8), 616-628 (2014).

40. Ramtvedt, B.E., Roinas, E., Aabech, H.S., Sundet, K.S. Clinical Gains from Including Both Dextroamphetamine and Methylphenidate in Stimulant Trials. Journal of Child and Adolescent Psychopharmacology. (2013).

41. Sangal, R.B., Sangal, J.M. Attention-deficit/hyperactivity disorder: using P300 topography to choose optimal treatment. Expert Review in Neurotherapy. 6 (10), 1429-1437 (2006).

42. Ogrim, G., Hestad, K.A., Brunner, J.F., Kropotov, J. Predicting acute side effects of stimulant medication in pediatric attention deficit/ hyperactivity disorder: data from quantitative electroencephalography, event-related potentials, and a continuous-performance test. Neuropsychiatric Disease and Treatment. 9, 1301-1309 (2013).

43. Barkley, R.A., DuPaul, G.J., McMurray, M.B. Attention deficit disorder with and without hyperactivity: clinical response to three dose levels of methylphenidate. Pediatrics. 87 (4), 519-531 (1991).

44. Chabot, R.J., Orgill, A.A., Crawford, G., Harris, M.J., Serfontein, G. Behavioral and electrophysiologic predictors of treatment response to stimulants in children with attention disorders. Journal of Child Neurology. 14 (6), 343-351 (1999).

45. Hale, J.B. et al. Executive impairment determines ADHD medication response: implications for academic achievement. Journal of Learning Disabilities. 44 (2), 196-212 (2011).

46. Tannock, R., Ickowicz, A., Schachar, R. Differential effects of methylphenidate on working memory in ADHD children with and without comorbid anxiety. Journal of the American Academy of Child and Adolescent Psychiatry. 34 (7), 886-896 (1995).

47. Ogrim, G., Aasen, I.E., Brunner, J.F. Single-dose effects on the P3no-go ERP component predict clinical response to stimulants in pediatric ADHD. Clinical Neurophysiology. 127 (10), 3277-3287 (2016).

48. Ogrim, G. et al. Predicting the clinical outcome of stimulant medication in pediatric attention-deficit/hyperactivity disorder: data from quantitative electroencephalography, event-related potentials, and a go/no-go test. Neuropsychiatric Disease and Treatment. 10, 231-242 (2014).

49. Ogrim, G., Kropotov, J.D. Predicting Clinical Gains and Side Effects of Stimulant Medication in Pediatric Attention-Deficit/Hyperactivity Disorder by Combining Measures From qEEG and ERPs in a Cued GO/NOGO Task. Clinical EEG and Neuroscience. 50 (1), $34-43$ (2019).

50. Lenartowicz, A., Loo, S.K. Use of EEG to diagnose ADHD. Current Psychiatry Report. 16 (11), 498-014-0498-0 (2014).

51. Johnstone, S.J., Barry, R.J., Clarke, A.R. Ten years on: a follow-up review of ERP research in attention-deficit/hyperactivity disorder. Clinical Neurophysiology. 124 (4), 644-657 (2013).

52. Gamma, A., Kara, O. Event-Related Potentials for Diagnosing Children and Adults With ADHD. Journal of Attention Disorders. (2016).

53. van Dinteren, R. et al. Utility of event-related potentials in predicting antidepressant treatment response: An iSPOT-D report. European Neuropsychopharmacology. 25 (11), 1981-1990 (2015) 